\title{
RECONSTRUCTIVE SURGERY OF UPPER EXTREMITY AFTER THERMAL BURNS - GUIDELINES OR EXPERIENCE
}

\author{
A. STRITAR MD PhD, M. Mikša MD, L. Emeršič MD, A. Arhar \\ Department of Plastic Surgery and Burns, University Medical Centre Ljubljana, Slovenia
}

\section{Objectives}

Hand burns hold a special place in the field of burn care, even though the surface area of the hand comprises approximately $1 \%$ TBSA. This is due to their anatomical, functional and aesthetic characteristics. Surgical treatment is a priority in these types of burns, especially on the dorsum of the hand and fingers.

\section{Methods}

Early tangential excision is the method of choice for treating deep dermal and subdermal hand burns. In these cases surgery yields considerably better functional and aesthetic outcomes, as opposed to conservative therapy. Consequently, hands are given priority over other parts of the body in surgical interventions.

Physiotherapy and rehabilitation play a very important role in the treatment of hand burns. Functionality of the hand is of paramount importance for the quality of life of burn patients. Some general rules apply for the treatment of hand burns, i.e. an individualised approach, careful patient selection and operative therapy based on the principles of the reconstructive ladder.

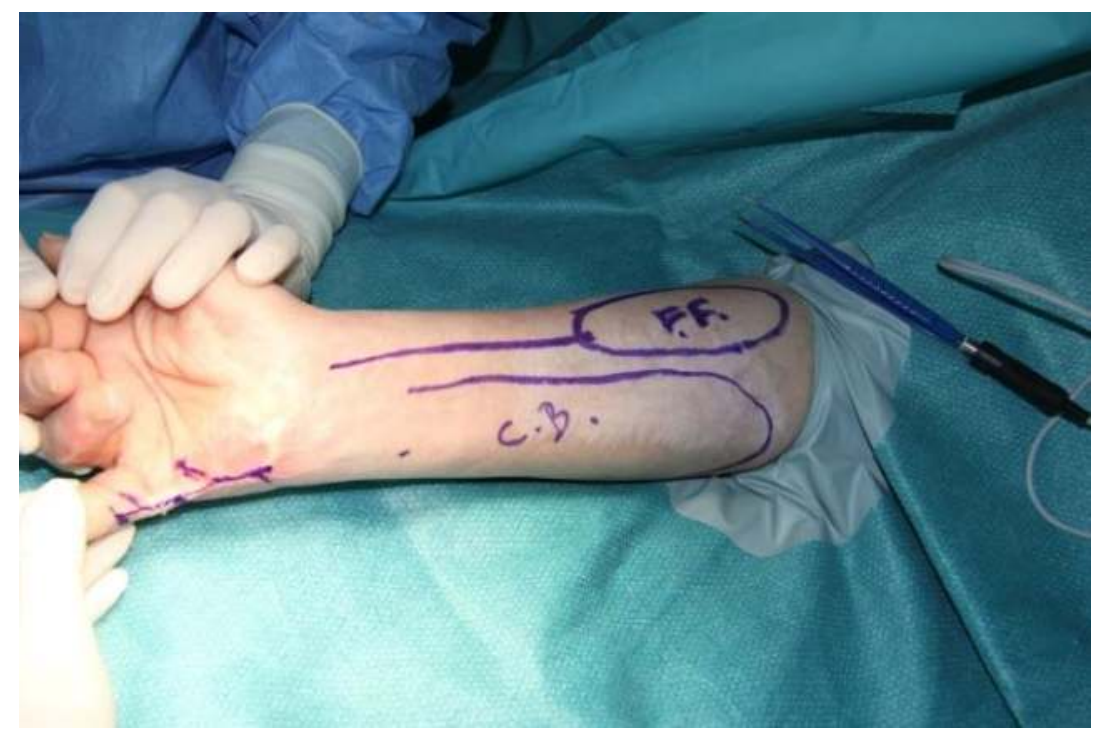

Fig. 1: Preoperative flap planning

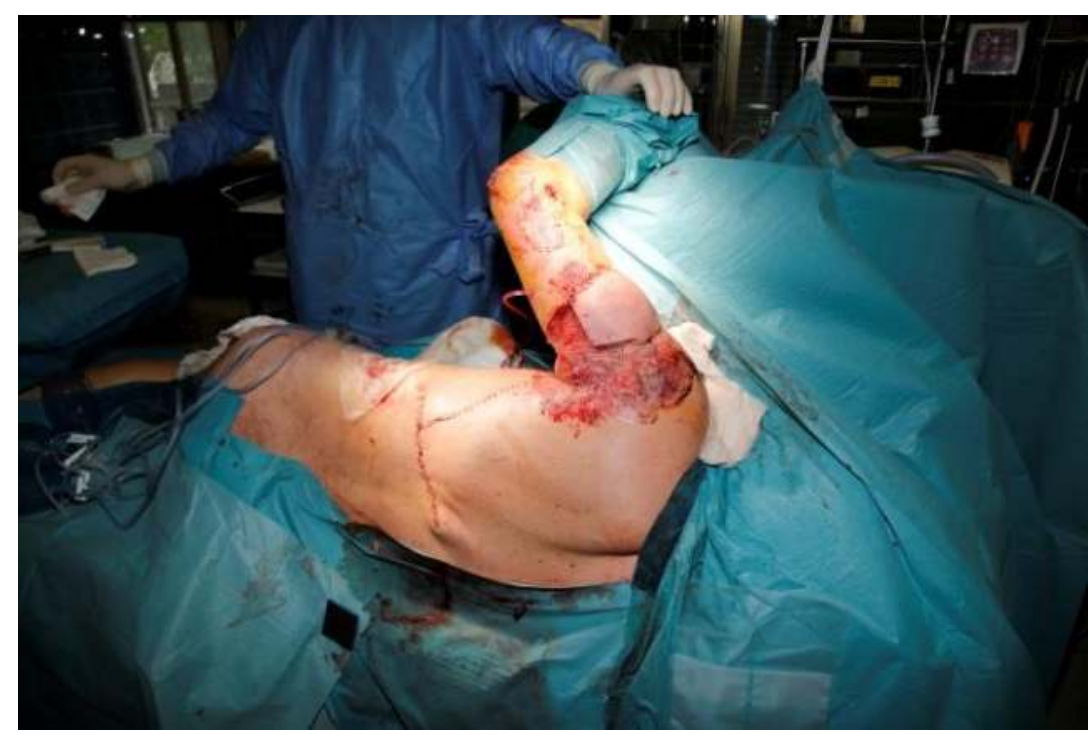

Fig. 4: Distant flap

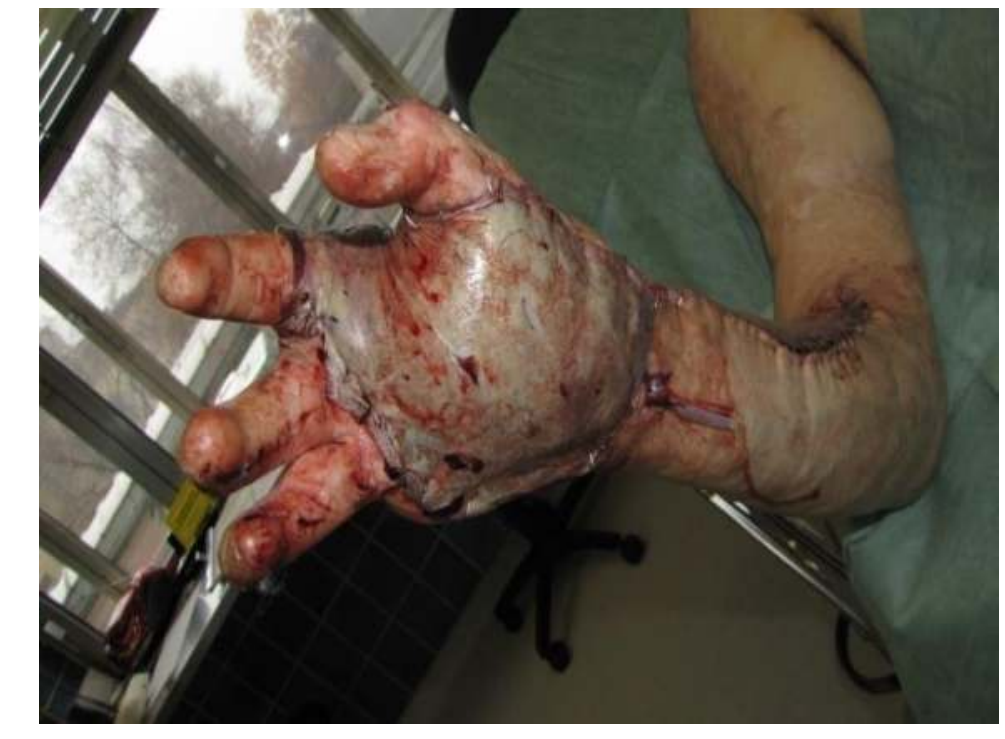

Fig. 2: Dislocated muscle flap

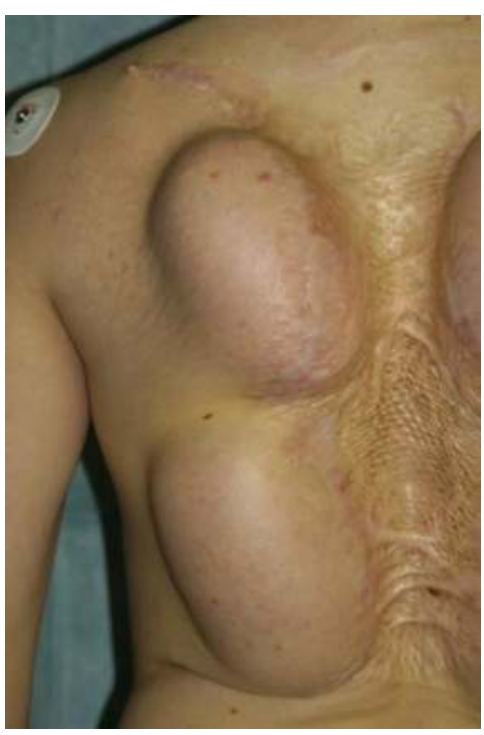

Fig. 5: Tissue expanders

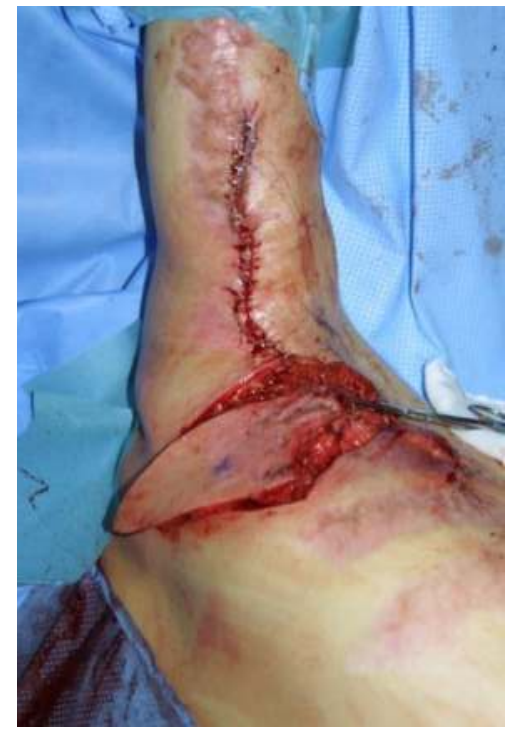

Fig. 3: Local perforator flap

\section{Results}

Patient's psychophysical state and participation are undoubtedly important; they have to be monitored in addition to providing the patient with objective information regarding their treatment. The treatment should be opportune and carefully planned in regard to the expected timeline as well as to the prediction of the end result. Postoperative follow-up is lengthy, several operations may be required and the rehabilitation programme completes the whole treatment.

\section{Conclusion}

Guidelines and experience occasionally differ. The former primarily protect logistic and forensic facts, while the latter expresses operative, surgical and patient's outcomes. Nevertheless, hand reconstruction, be it primary or late, should abide by the protocol without sudden changes or improvisation, while being in accordance with the expected timeline and burn scar maturation. 